\title{
La autoevaluación institucional y su incidencia en las prácticas pedagógicas ${ }^{1}$
}

\author{
JohnNy VARgas ZULUAGa ${ }^{2}$
}

\begin{abstract}
Resumen
La investigación se realizó en la institución educativa Nuestra Señora del Rosario del municipio de Villamaría (Caldas) y busco comprender los factores que inciden en las prácticas pedagógicas, a partir de los procesos de autoevaluación institucional.

Los hallazgos de esta investigación permiten valorar los resultados de la autoevaluación, para construir nuevos significados en la práctica pedagógica de los docentes en la institución, donde convergen múltiples factores desde la gestión administrativa, pedagógica, curricular y comunitaria. En este sentido se considera que la evaluación institucional tiene concepciones teóricas que inciden en las prácticas pedagógicas y son generadoras de procesos de debate en relación con la calidad educativa.

El Ministerio de Educación Nacional formula lineamientos que ayudan a las instituciones educativas del país, a plantear alternativas y soluciones a los problemas e interrogantes que se presentan en el contexto, con la participación de actores locales y así abordar nuevas relaciones entre la formación del maestro, la calidad educativa, los desempeños de los estudiantes y practicas pedagógicas que se imprimen en un plan de mejoramiento vigente durante el periodo escolar.

Palabras clave: Educación, formación, calidad, cultura, política, contexto, escuela.
\end{abstract}

\section{Abstract \\ Institutional self-evaluation and its impact on teaching practices}

This research was conducted at the educational institution Our Lady of the Rosary of the municipality of Villamaria (Caldas) and sought understanding of the factors that affect teaching practices, from the processes of institutional self-assessment.

The findings of this research to evaluate the results of the self, to construct new meanings in the pedagogic practice of teachers in the institution, where multiple factors converge from the administrative management, pedagogical, curricular, and community. In this sense it is considered that the institutional assessment has theoretical conceptions that have an impact on teaching practices and processes are generating discussion in relation to the quality of education.

1 Recibido: 03 de Mayo de 2015. Aceptado: 06 de junio de 2016.

2 Magister en educación docencia de la Universidad de Manizales; Licenciado en educación Física, recreación y deportes de la universidad de Caldas. Correo electrónico: jvargasz.72@hotmail.com 
The Ministry of National Education formula guidelines to help educational institutions in the country, to suggest alternatives and solutions to the problems and questions that are presented in context, with the participation of local actors to address new relationships between the teacher training, educational quality, the performances of the students and teaching practices that are printed on an improvement plan in force during the school term. Keywords: Education, training, quality, culture, politics, context, school.

\section{Introducción}

Pensar lo impensado, develar lo velado es un acto inlocutivo, autónomo desde lo estético y lo ético, implica otear la armonía del universo renacer cada día, como lo hace la esperanza con la aurora, sentir plenamente el pasado, el presente y el futuro embriagándonos con las realidades pletóricas, místicas que encierran nuestro mundo en un acto de sueño en conciencia que provoque una renovación de los saberes epistémicos. Molina, G. (2008, p. 190)

La época actual es de profundos cambios, inmersos en la vida cotidiana que dan una radical perspectiva frente a la educación, la cultura y la tecnología. Las instituciones educativas parten del hecho de reconocer como desarrollan su labor educativa, para así inferir la capacidad de formar ciudadanos que respondan a los desafíos del presente. El mejoramiento de las instituciones educativas es un reto y desafío permanente para mirar la realidad de la formación de sus actores, mejorar el proceso de formación, construir su historia y atender a las problemáticas desde el marco contextual, legal y cultural donde los conceptos de hombre, educación, cultura son criterios de la política de formación y se presentan en estructuras organizativas para la gestión escolar, donde las improntas pedagógicas, curriculares y didácticas son alternativas que se adecuan a las condiciones de los territorios vitales, existenciales y geográficos donde está inmerso los proyectos educativos (Amador, 2007). El espíritu de los planes de mejoramiento de las instituciones educativas se enmarcan en la constitución política de 1991, la ley 115 de 1994 general de educación, donde el sentido es buscar y crear las condiciones que permitan ofrecer educación acorde con las exigencias políticas, culturales y sociales basados en los principios de la democracia, participación y autonomía.

Los planes de mejoramiento educativo establecen balances en las fortalezas y oportunidades del desarrollo institucional, desde las concepciones y prácticas asumidas por los docentes en torno a la evaluación institucional. Reconoce la parte subjetiva de los actores en la práctica profesional y en el contexto institucional.

\section{La evaluación un asunto de todos}

La evaluación evoluciona de acuerdo con las tendencias en los procesos de desarrollo social, económico y cultural en los países; a nivel regional la preocupación por lograr la calidad educativa ha ampliado su espectro, se evalúa el conocimiento de los actores participantes del proceso educativo y la evaluación institucional desde las gestiones académicas, administrativas, pedagógicas y comunitarias.

La evaluación es una herramienta de gestión que permite establecer estándares de calidad y conocer que tan lejos estamos de que los niños y jóvenes aprendan lo que deben aprender. Es un hecho comprobado que las prácticas administrativas que lo que se mide mejora. Conocer los 
resultados permite a la institución revisar sus prácticas pedagógicas y establecer los procesos de mejoramiento. (Secretaria de Educación de Bogotá, 2001, p. 1)

La evaluación es un asunto de todos y sirve para todos, permite a la institución educativa reorganizar conceptos, unificar criterios, configurar prácticas, tomar decisiones, redimensionar procesos y priorizar acciones frente a la formación de los educandos, la actuación de los docentes y directivos y dar sentido de la educación para las familias y la comunidad.

\section{Perspectiva crítica $y$ evaluación de la educación}

Las instituciones educativas están en el dilema de pensarse así mismas, pero el carácter indeterminado de la economía, el conocimiento y la cultura generan condiciones políticas y sociales que inciden en las formas culturales de su organización y gestión. Las instituciones educativas como identidades sociales han sido estructuradas por el modelo de desarrollo económico con implicaciones en los procesos de formación.

Entender la construcción institucional en el marco de la educación contemporánea, tiene variedad de criterios; que requieren comprender los niveles sociales en que se moviliza la educación; el nivel macro desde la política y la economía, el nivel micro desde las comunidades y el nivel meso desde la organización institucional. Este es un acercamiento en perspectiva crítica de constante interacción donde el proceso educativo y sus maneras determinan intereses que implican la formación y la disposición de las gestiones institucionales.

\section{La duda}

El tema de la autoevaluación institucional tiene una amplia variedad de trabajos de investigación (Martín, Michaela (2002), Zuluaga, Olga (2005), Gonzales, Diego (2011), Cumaco, Ricardo $(2011), \ldots)$ lo que da origen a la diversidad de teorías e interpretaciones, enmarcadas en autoevaluación, autorregulación y acreditación como elementos inherentes a los procesos de mejoramiento y calidad.

Se considera que la autoevaluación juega un papel potenciador en el éxito académico, en los ambientes institucionales y en el desempeño de mejoramiento de las prácticas docentes. Por ello el bajo rendimiento académico de los estudiantes ha sido relacionado con actitudes del poco interés hacia el estudio, la participación en clase, la asistencia, la puntualidad y el cumplimiento de actividades asignadas en las diferentes áreas, lo que está relacionado con los propósitos formativos, desde la institución y las practicas pedagógicas de los docentes.

Estas situaciones se relacionan con el contexto en el cual está ubicada la institución educativa, y con la valoración social que se tiene desde el proyecto educativo institucional (PEI). El contexto se caracteriza por la presencia del Estado que ha instituido la política educativa en aspectos, tales como: la ampliación de cobertura, la inclusión, las relaciones maestro-alumno, los planes de autoevaluación, los planes de mejoramiento y el seguimiento orientados a la calidad educativa, los cuales se convierten en un medio para mantener la estructura social del estado y reproducir los intereses políticos y económicos.

En virtud de lo anterior existen multiplicidad de factores que implican el asunto de la calidad educativa y ante la preocupación de los procesos de autoevaluación institucional, surge la pregunta para afrontar caminos de desarrollo educativo: ¿Qué impacto tienen los resultados de la autoevaluación institucional en el mejoramiento de las prácticas pedagógicas en las instituciones educativas? (En el estudio de la Institución Educativa Nuestra Señora del Rosario del municipio de Villamaría - Caldas).

Uno de los temas preocupantes de nuestro sistema educativo es la eva- 
luación, entendida como un medio y no como finalidad, es deber de los docentes implicarnos en los procesos de calidad educativa desde las prácticas docentes, para responder a los cambios y necesidades de la sociedad e intervenir en la crisis del sistema.

La autoevaluación institucional desarrolla un papel protagónico para inferir procesos de mejoramiento en la formación de los sujetos, amplía su alcance y responsabilidad de su ejercicio, libera la duda de cómo se ven afectadas las practicas pedagógicas de los docentes, en el marco de acción cotidiana de la formación.

La construcción y la trasformación social, desde la autoevaluación institucional, perciben mecanismos de interpretación y reflexión individual para ser potenciadas en la formación del estudiante e incidir en la calidad educativa.

\section{Cuestión esencial}

Las lecturas a la política educativa Colombiana, enmarcadas desde los planes de desarrollo municipal, la política nacional definida en el plan sectorial de Educación Nacional "Educación de Calidad, el camino para la prosperidad", Visión Colombia 2019, las líneas misionales de Cobertura, Calidad, Pertinencia y Eficiencia, propositivos del contexto internacional, y los consensos construidos en el escenario del Pacto social por la Educación (Plan Decenal Nacional 2006-2016), enfatizan en la idea histórica de este proceso: autoevaluación institucional con una mirada política y administrativa, que devela la realidad del cómo se optimizan los recursos (cobertura y resultado) y las inversiones en el sector, con una impronta de mejoramiento de la calidad.

En los últimos 20 años en Colombia se acentúa la política de mejoramiento de la Calidad para valorar el nivel que poseen las instituciones educativas de carácter público y privado. Implementa normas jurídicas que se expresan en leyes y decretos, para incidir en la reforma política, pedagógica, didáctica y curricular en las instituciones educativas en indicadores de cobertura, situación que deja de lado las lecturas de corte cualitativo en satisfacciones, deseos e implicancias en el desarrollo humano y social cuestión esencial en la evaluación.

\section{Tejido de la autoevaluación institucional}

\section{¿La gestión educativa en Colombia, un tejido de la norma al ser de la evaluación?}

El Ministerio de Educación Nacional como estamento de dirección educativa, promulga su política y la disemina en normas y documentos que dan orientación para su desarrollo.

Desde el marco constitucional Colombiano de 1991 y en desarrollo del artículo 67 se expresa:

La educación es un derecho de la persona y un servicio público que tiene una función social: con ella se busca el acceso al conocimiento, a la ciencia, a la técnica, y a los demás bienes y valores de la cultura.

La educación formara al colombiano en el respeto a los derechos humanos, a la paz y a la democracia; y en la práctica del trabajo y la recreación, para el mejoramiento cultural, científico, tecnológico y para la protección del ambiente.

El Estado, la sociedad y la familia son responsables de la educación, que será obligatoria entre los cinco y los quince años de edad y que comprenderá como mínimo, un año de preescolar y nueve de educación básica. La educación será gratuita en las instituciones del Estado, sin perjuicio del cobro de derechos académicos a quienes puedan sufragarlos. Corresponde al Estado regular y ejercer la suprema inspección y vigilancia de la educación con el fin de velar por su calidad, por el cumplimiento de sus fines y por la 
mejor formación moral, intelectual y física de los educandos; garantizar el adecuado cubrimiento del servicio y asegurar a los menores las condiciones necesarias para su acceso y permanencia en el sistema educativo.

La Nación y las entidades territoriales participarán en la dirección, financiación y administración de los servicios educativos estatales, en los términos que señalen la Constitución y la ley.

Se promulga la ley 115 general de educación en el año de 1994, el decreto 1860 en referencia a los Proyectos Educativos Institucionales, que dan orientación para el servicio educativo y la construcción de un proyecto político de nación.

En esta dirección como proceso de mejoramiento y desarrollo de la política educativa, se realizan transformaciones desde las competencias y recursos para el sector social y se emana la ley $715 \mathrm{del}$ 21 de diciembre de 2001, "Por la cual se dictan normas orgánicas en materia de recursos y competencias de conformidad con los artículos 151, 288, 356 y 357 (Acto Legislativo 01 de 2001)" reforma que condiciona la reorganización de los sectores en proceso y resultado y por consiguiente en impacto de sus coberturas y calidades, tanto en educación como en salud.

Este tejido normativo y jurídico promueve la evaluación cuantitativa y la gestiona en los sectores de educación y salud. En el tejido educativo se despliegan documentos y lineamientos de política como son las guías que emana el Ministerio de Educación Nacional, que están ligadas a los procesos denominados de reorganización educativa, que consiste en la racionalización de la planta de personal docente, la fusión de los establecimientos educativos.

En el año 2003, el Ministerio de Educación Nacional, da a conocer la guía No. 11, "Guía de autoevaluación para el mejoramiento institucional", para orientar los procesos de autoevaluación y mejoramiento institucional, ella configura el esquema de cuatro gestiones institucionales: Gestión directiva, Gestión académica, Gestión administrativa Financiera y la Gestión Comunitaria.

Posteriormente se emana la guía 34 "De la autoevaluación al mejoramiento" agrupa los procesos en la gestión escolar, reseña el concepto de calidad en educación a partir de planes de mejoramiento.

Conceptos de autonomía escolar, participación comunitaria, integración institucional y democracia reorganizan el imaginario de la gestión escolar, que se mediatiza en instrumentos que dan cuenta de coberturas y resultados, e infieren calidad y mejoramiento. Es fundamental comprensiones en la dimensionalidad ética y política de la gestión escolar para los procesos educativos y formativos de la sociedad, en visiones de desarrollo humano y social.

En virtud de lo anterior el estudio propone valorar el impacto de los resultados de la autoevaluación institucional y develar las concepciones y prácticas que construyen los docentes acerca de la autoevaluación institucional. Este planteamiento lo afronta la escuela que ha de transformar los procesos frente a indagar si se conocen las problemáticas que se viven en el contexto si se han abordado estrategias para establecer posibles soluciones.

\section{La autoevaluación institucional}

La autoevaluación institucional, es un proceso más de la gestión escolar en la cual se dimensiona el concepto educativo, pedagógico, didáctico y curricular, cuya acción está en permanente mejora y obedece a las condiciones y necesidades de la formación.

La autoevaluación obedece a una postura ética y en función de principios de:

- Veracidad: En el acto de educación se hacen las cosas bien.

- Corresponsabilidad: En el acto de educación se comprometen acciones y es de esfuerzo colectivo. 
- Participación: En el acto de educación se parte del conocimiento y análisis de condiciones que implican a los actores comunitarios.

- Continuidad: En el acto de educación se identifican procesos y oportunidades que se operan en acciones de mejoramiento.

Así el acto de educación es un acto de pensamiento, conocimiento y sensibilidad frente a la formación humana. Ese acto educativo implica potenciar las capacidades, para la auto liberación, auto determinación para posibilitar la autonomía del sujeto. La autoevaluación se centra en el posicionamiento antropológico, donde el hombre como ser cultural e histórico desarrolla la capacidad para crear cultura y recrearse en sí mismo y afianzar la identidad, el posicionamiento político para definir la autonomía y actuación social, el posicionamiento epistemológico en la claridad sobre el carácter activo y transformativo del conocimiento y sus condiciones de posibilidad, el posicionamiento cultural y social que infiere comprender las relaciones hombre-sociedad.

\section{Gestión educativa}

La gestión educativa asume los procesos y acciones para la formación de actores en contexto determinado. Se fundamenta en el reconocimiento constitucional de la educación como derecho y como servicio público con función social. Se ejerce desde la suprema vigilancia para velar por su calidad, por sus finalidades y la formación moral, física, cognitiva e intelectual de los educandos y en garantía de prestación del servicio y aseguramiento de condiciones de acceso y permanencia en el sistema.

La gestión educativa reconoce en la educación los propósitos de formación del talento humano en perspectiva de derechos. Así la gestión educativa asume las finalidades del sistema educativo colombiano, como formación del capital social, que se expresan en la ley general de educación 115 de 1994 articulo 5, que transitan por el pleno desarrollo de la personalidad, el derecho a la vida, la formación en el respeto a la cultura e historia Colombiana, el acceso y generación de conocimientos científicos y técnicos al igual que el desarrollo de la capacidad crítica y reflexiva de las personas; para la creación y apropiación de habilidades, actitudes, capacidades y hábitos intelectuales para el desarrollo del saber.

La gestión educativa incorpora la visión social y la visión humana en una perspectiva de calidad, en huella de la persona y su personalidad para efectos en la dimensionalidad social.

\section{Metodología de la investigación}

El estudio se enmarca en la investigación cualitativa ${ }^{3}$ y su perspectiva se orienta hacia la gestión escolar (la acción y el cambio) para cualificar y transformar la labor docente y directiva docente e intervenir los problemas de la institución educativa, desde planes de mejoramiento.

El tipo de estudio es descriptivo dado el interés de identificar las prevalencias existentes en la institución educativa NUESTRA SEÑORA DEL ROSARIO del municipio de Villamaría (Caldas), y evidenciarlas en el plan de mejoramiento. La descripción del proceso educativo en perspectiva de calidad, en el contexto Colombiano, contribuye a comprender la realidad de la gestión escolar y obtener una visión amplia "la descripción prepara el paso a la explicación por medio de la cual de aclara y se hace comprender la información recolectada" (Cerda, 2005, p. 72)

Taylor y Bogdan (1992) define la metodología como la manera de enfocarnos a

3 Proceso no matemático de interpretación, realizado con el propósito de descubrir conceptos y relaciones en los datos brutos y luego organizarlos en un esquema explicativo teórico (Corbin \& Strauss, 2002, p.12). 
los problemas, como a la forma de buscar respuestas en los mismos, Guba (1990) permite diferenciar los enfoques o paradigmas de investigación social, a partir de la respuesta a preguntas ¿Cómo se concibe la naturaleza tanto del Conocimiento como de la realidad?, ¿Cómo se concibe la naturaleza de las relaciones entre el investigador y el conocimiento que genera?, ¿Cuál es el modo en que construye o desarrolla conocimiento el investigador?

El estudio argumenta su análisis en la descripción que se instaura en la posibilidad de la comprensión de la gestión escolar, conocer su sentido, identificar las relaciones que existen en lo administrativo, pedagógico y curricular. Para lograr comprensión se hace necesario estructurar el referente de comprensión histórica de la gestión escolar y su mirada de calidad, que se dimensiona en los planes de mejoramiento. Se da un dialogo entre el fenómeno de estudio y los actores de indagación en la realidad contextual para obtener la visión integral del objeto de estudio.

\section{Proceso metodológico}

La investigación es cualitativa de tipo descriptivo y transita por momentos, así:

Momento Uno, Análisis documental: en documentos constitución política de Colombia, ley general de educación 115 de 1994. Decretos de evaluación 1860 de 1994,0230 del 11 de febrero de 2002,1290 de abril 16 de 2009 y la Guía "De la autoevaluación al mejoramiento" Numero 34 y la guía no 11 "Guía de autoevaluación para el mejoramiento institucional".

Momento Dos, Descriptivo: Se realiza lectura del proyecto educativo institucional sus propósitos formativos, se centra el interés en la observación de la gestión administrativa, académica y comunitaria, se constata la presencia o prevalencia de los procesos de gestión, se establecen relaciones para hacer el diagnostico sobre la situación actual de la institución en cumplimiento de objetivos y metas de calidad académica desde el desarrollo del plan de estudios. En este proceso la institución educativa recoge, sistematiza y valora la información y la compara con los resultados en las áreas de gestión para sugerir elementos en el plan de mejoramiento institucional. La información se recoge desde los documentos institucionales y la conformación de grupos focales.

Momento tres, Análisis y alcances de conocimiento: a partir de la descripción y contextualización, se toman las categorías del estudio: autoevaluación institucional, normativa política y prácticas pedagógicas. Y se refleja la particularidad de la institución de carácter público y la incidencia de la gestión en los planes de mejoramiento.

\section{Resultados y discusión}

Con la recolección de información a través de los grupos focales y encuestas realizadas a los académicos de directivos y docentes, se categoriza la información recopilada en un esquema explicativo, que permitió ampliar la comprensión teórica existente de la gestión escolar en el marco de calidad educativa. Se realizo transcripción de la información y posteriormente se desarrolla el proceso de categorización.

\section{El análisis de información:}

Los directivos cumplen lo establecido en la guía 34 del Ministerio de educación Nacional de manera operativa, desde lo político generan espacios de discusión frente a los resultados encontrados en la autoevaluación, situación descrita y confrontada a partir del instrumento de encuesta que tuvo las siguientes preguntas (Aplicada a directivos y docentes, ficha técnica de encuesta):

- ¿La institución educativa genera mecanismos para socializar el contenido de la guía 34 de autoevaluación institucional? 
- ¿La autoevaluación institucional se realiza de acuerdo a lo establecido en la guía?

- ¿En el proceso de autoevaluación institucional se valoran los distintos procesos de la gestión educativa (directiva, académica, administrativa y de comunidad) Participa usted valorando las cuatro gestiones de la autoevaluación?

- ¿En el proceso de autoevaluación institucional participa los diferentes actores de la comunidad educativa?

- ¿considera que el instrumento que se emplea para realizar la autoevaluación institucional es pertinente $y$ coherente?

- ¿La información resultado del proceso de autoevaluación institucional sirve de insumo para la elaboración de los planes de mejoramiento?

- ¿Los planes de mejoramiento son socializados en el momento oportuno y se les hace el respectivo seguimiento?

- ¿En la construcción del plan de mejoramiento institucional, se abren espacios de participación a los estudiantes y padres de familia?

- ¿los planes de mejoramiento institucional permiten mejorar el rendimiento y desempeño en el aula de clase?

- ¿la autoevaluación institucional afecta las practicas pedagógicas en el aula de clase?

Donde las respuestas tenían las siguientes escala:

\begin{tabular}{|l|l|}
\hline Siempre & \\
\hline Algunas veces & \\
\hline Nunca & \\
\hline
\end{tabular}
así:

Y generando resultados en porcentaje

\begin{tabular}{|c|c|c|c|c|}
\hline Encuesta & $\mathbf{N}^{0}$ pregunta & Siempre & Algunas veces & Nunca \\
\hline \multirow{4}{*}{$\begin{array}{c}\text { Numero de Directivos } \\
\text { Docentes (2) }\end{array}$} & 1 & $50 \%$ & $50 \%$ & $0 \%$ \\
\cline { 2 - 5 } & 2 & $100 \%$ & $0 \%$ & $0 \%$ \\
\cline { 2 - 5 } & 3 & $0 \%$ & $100 \%$ & $0 \%$ \\
\cline { 2 - 5 } & 4 & $0 \%$ & $100 \%$ & $0 \%$ \\
\cline { 2 - 5 } & 5 & $100 \%$ & $0 \%$ & $0 \%$ \\
\cline { 2 - 5 } & 7 & $0 \%$ & $100 \%$ & $0 \%$ \\
\cline { 2 - 5 } & 8 & $50 \%$ & $100 \%$ & $0 \%$ \\
\cline { 2 - 5 } & 9 & $100 \%$ & $50 \%$ & $0 \%$ \\
\hline
\end{tabular}

\begin{tabular}{|c|l|l|l|l|}
\hline Encuesta & \multicolumn{1}{|c|}{$\mathbf{N}^{0}$ pregunta } & \multicolumn{1}{|c|}{ Siempre } & \multicolumn{1}{|c|}{ Algunas veces } & \multicolumn{1}{c|}{ Nunca } \\
\hline \multirow{5}{*}{$\begin{array}{c}\text { Número de Docentes } \\
(15)\end{array}$} & 1 & $66.6 \%$ & $33.4 \%$ & $0 \%$ \\
\cline { 2 - 5 } & 2 & $93.7 \%$ & $6.3 \%$ & $0 \%$ \\
\cline { 2 - 5 } & 3 & $53.3 \%$ & $20 \%$ & $26.7 \%$ \\
\cline { 2 - 5 } & 4 & $26.7 \%$ & $33.3 \%$ & $40 \%$ \\
\cline { 2 - 5 } & 5 & $86.6 \%$ & $13.4 \%$ & $0 \%$ \\
\cline { 2 - 5 } & 7 & $60 \%$ & $40 \%$ & $0 \%$ \\
\cline { 2 - 5 } & 8 & $26.6 \%$ & $53.4 \%$ & $20 \%$ \\
\cline { 2 - 5 } & 9 & $26.6 \%$ & $40 \%$ & $33.4 \%$ \\
\cline { 2 - 5 } & 10 & $53.3 \%$ & $46.7 \%$ & $0 \%$ \\
\hline
\end{tabular}


Dichos resultados evidencian que:

\section{Los Directivos:}

- Los directivos cumplen lo establecido en la guía 34 del Ministerio de educación Nacional, con conocimiento pero "sin reflexión y debate frente a los resultados que se pretenden alcanzar".

- Los resultados sirven para generar planes de mejoramiento en las cuatro gestiones.

- Se hacen seguimiento a los planes pero fluctúan en la respuesta, lo que evidencia que algunos resultados tienen seguimiento y otros no.

- La respuesta nunca no aparece afirmada en la encuesta

- Las preguntas sobre política y autoevaluación institucional son diversas y el mayor porcentaje está en "siempre" lo que corrobora la aplicación de la política sin reflexión.

\section{Los Docentes:}

- Un alto porcentaje de los docentes sostiene que se generan los espacios para realizar la autoevaluación institucional.

- En su gran mayoría los docentes consideran pertinente el uso de la guía 34 como modelo para realizar el proceso de autoevaluación.

- Según los resultados de la encuesta la autoevaluación no genera procesos de transformación de las prácticas pedagógicas.

- La Institución no genera la participación de todos los estamentos de la comunidad en los procesos de autoevaluación institucional.

- Los planes de mejoramiento no son socializados y tampoco se les hace el seguimiento oportuno.

- En el diseño de los planes de mejoramiento no participan todos los estamentos de la comunidad escolar.

- No se evidencia que se haga una autoevaluación completa de las 4 áreas de gestión.

- Un porcentaje considerable de docentes manifiesta que los resultados de la autoevaluación no se toman como un insumo para el diseño de los planes de mejoramiento.

Es así como los resultados proporcionan elementos fundantes que sirven para generar planes de mejoramiento en las instituciones en sus 4 gestiones, donde se puede concluir que la educación es un resultado planeado y permite el análisis desde el cumplimiento de indicadores, es decir eficacia. También se evalúa el impacto en su efectividad, donde los actores y su gestión demuestran responsabilidad en la permanencia, desarrollo y continuidad de lo educativo.

La gestión educativa asume los procesos y acciones para la formación de actores en contexto determinado. Se fundamenta en el reconocimiento constitucional de la educación como derecho y como servicio público con función social.

La gestión educativa en su desarrollo opera en planes de mejoramiento, que incluye el significado, sentido e intencionalidad de la educación en el contexto escolar, con requerimientos mínimos y condiciones de posibilidad, en finalidades educativas expresas en objetivos y metas, en componentes de transformación evidentes en los procesos curriculares, en componentes de orientación y sostenimiento del sistema de formación, evidentes en los procesos administrativos y en componente de control, seguimiento y perfeccionamiento, evidente en los procesos evaluativos.

La formación de los docentes y directivos docentes son preocupación para asumir la gestión escolar y generar cambio, innovación, mejoramiento y renovación de las prácticas docentes conducentes a mejorar la calidad de la educación, no obstante los procesos de formación no siempre producen los efectos esperados para imprimir sentido y coherencia a la formación de los educandos. Existe discrepancia en la expectativa de formación escolar y los procesos pedagógicos con 
criterio de aprendizaje intelectual significativo para actuar en sociedad.

La renovación de la docencia y las necesidades de modernización y cambio en las instituciones de formación en la educación inicial, básica y media son dispositivo necesario frente a la nueva dinámica de ciencia, tecnología, innovación y alternatividad para la formación. Se reconoce los procesos pero las docencias continúan siendo de tipo tradicional y discursiva.

La transformación en la gestión educativa exige un gran compromiso ético, social para promover la formación política en visión de derechos humanos, convivencia e identidad nacional. El sistema de producción de conocimiento a cambiado y se requiere la orientación de los currículos, las formas didácticas y la animación pedagógica para la formación de los sujetos en su desarrollo de habilidades para la vida.

Los cambios tecnológicos generan velocidad e incertidumbre frente al conocimiento y da incidencia en la gestión educativa para incorporar nuevas maneras de comunicar y desarrollar el aprendizaje, para la población del nuevo tiempo.

\section{Recomendaciones}

La autoevaluación institucional incide en las prácticas pedagógicas y se convierte en una cultura, implica todo un desafío para la institución y sobre todo permite superar las resistencias, las miradas, los obstáculos, expresos y construidos desde la comunidad. La autoevaluación incorpora proceso y experiencia de flexibilidad académica, pedagógica y curricular, por ello la recomendación está en la sistematización y coherencia en bien de los procesos de formación.

La organización de los actores educativos en la dinámica institucional permite la posibilidad de desarrollar prácticas de articulación y dinámicas de procesos en apertura, innovación y cambio que conllevan a nuevas formas y maneras de desarrollar la cultura institucional.

La investigación es un elemento importante y trascendente en la gestión institucional, donde permite la comprensión de asuntos cruciales de formación desde la perspectiva académica, pedagógica, curricular y comunitaria, y centra potencialidades hacia los desempeños para una formación centrada en capacidades, habilidades y destrezas hacia la vida.

\section{Bibliografía}

Amador, L; Arias, G; Cardona, S; García, L; Tobón, G. (2011). Educación, sociedad y cultura. Manizales: Ed. Universidad Católica de Manizales.

Amador, L. (2007). Formación en tiempos presentes hacia pedagogías emergentes. Revista Latinoamericana de Estudios Educativos, vol. 3, no 1, p. 41-63. Manizales: Universidad de Caldas

Barbier, M J. (1993). la evaluación en los procesos de formación, p. 31-167 Barcelona: Paidós Ibérica

Calvo, G; Rendón, D y García, L. (2010). Un diagnóstico de la formación docente en Colombia. IESALC de la UNESCO. Bogotá: IESLAC
Cerda, H. (2005). Los elementos de la investigación. Santa fe de Bogotá: El búho.

Cornejo, R. (2003). Trabajo docente en la Institución Educativa. [Documento base: Programa de doctorado en Sicología]. Santiago de Chile: Universidad de Chile.

Cumaco, R. (2011). La transversalidad curricular: conceptos, prácticas y rupturas para el departamento del Huila. Tesis de maestría en educación. Manizales: Universidad de Caldas.

Equipo de Calidad PEI (2011) Institución Educativa Nuestra Señora Del Rosario, $p$. 56-73. Villamaría, Caldas (No publicado)

Equipo De Calidad PEI. (2011). Institución Educativa Nuestra Señora Del Rosario sis- 
tema de evaluación y promoción. Villamaria, Caldas (No publicado)

Escobar, N. (2007). Práctica profesional docente desde la perspectiva de los practicantes y tutores. Revista Acción Pedagógica, $N^{\circ}$ 16 / Enero - Diciembre, 2007 - p. 182- 193

Foucault, M. (1988). El sujeto y el poder. Revista Mexicana de Sociología, Vol. 50, No. 3. (Jul. - Sep) pp. 3-20. México: Universidad Nacional Autónoma de México.

Freire, P; Castell, M; Flecha, R; Giroux, H; Macedo D y Willis, P. (1997). Nuevas perspectivas críticas en educación, jóvenes diferencia y educación postmoderna, Primera edición. Buenos aires: Paidós.

Gonzales, D. (2011). La apropiación de aspectos motivacionales como estimulo para lograr la eficacia, la eficiencia y la efectividad en la gestión institucional, un análisis desde los docentes de la Institución Educativa Liceo Quindío. Tesis de maestría en educación. Manizales: Universidad de Caldas

González González, M A. (2009) Horizontes humanos límites y paisajes. Manizales: Editorial Universidad de Manizales, ISBN: 978958-9314-49-4.

González González, M A. (2016). El Genocidio armenio, humillación a un grupo humano al delirar la razón ilustrada: cien años de soledad y apatía. En: Ghukasian, A (Coord), Cien Años del Genocidio armenio: un siglo de silencio (pp. 83-96). Castellón de la Plana: e-DitARX Publicaciones digitales.

Guba, E. (1990). The Paradigm Dialogic. California: Sage.

López de Maturana, S. (2010). Las complejidades emergentes en las historias de vida Los buenos profesores. Revista de la Universidad Bolivariana, Volumen $9, \mathrm{~N}^{\circ} 25$, 2010, p. 255-267

Magni, R. (2006). Rol docente en el tercer milenio. En: http://www.educar.org/articulos/ roldocente.htm (Recuperado el 08 de agosto de 2012)
Martín, M, and Paula R. (2002). Estudios de caso sobre acreditación en Colombia, Hungría, India, Filipinas y Estados Unidos: tan similares pero tan diferentes. Primer Seminario Internacional: Educación superior, calidad y acreditación. Santafé de Bogotá: Consejo Nacional de Acreditación equidad y la convivencia. Plan Decenal de Educación (2002).

Mclaren, P. (1984). Una introducción a la pedagogía critica en los fundamentos de la educación. Primera edición en español. Siglo XXI editores, 1984. pp. 203-234

Ministerio de Educación República de Colombia (1994) Ley general de educación y sus decretos reglamentarios 1994

Ministerio de Educación República de Colombia decreto ley 1290 de 2009

Ministerio de Educación República de Colombia (2002) Guía 34 para el mejoramiento institucional Título: Carta de la ministra de educación nacional Colombia p. 07-09

Ministerio del Interior (1991) República de Colombia Derecho a la Educación y al trato Digno 1991

Molina, G. (2008). Hacia la construcción de una evaluación no parametral en el horizonte del siglo XXI. Una mirada desde la estética. Revista Plumilla Educativa Nro. 6. Manizales: Universidad de Manizales.

Quintero, P. (2001). Aproximación a las practicas pedagógicas a universidad del valle sede zarzal. 2001 pp 74. Universidad del Valle.

Villar, A. (2004). Hacia una formación de docentes competentes, que posibilite la práctica pedagógica autónoma investigativa, como elemento articulador del profesional de la educación. Colectivo de Investigación en Matemática Educativa CIMES. Madrid: Ed. Morata

Zuluaga, O. (2005). Una lectura desde la práctica pedagógica. En: Zuluaga et. alt. La pedología y la Educación. Bogotá: Cooperativa Editorial Magisterio 